\title{
Observations et recherches expérimentales sur la variation chez le Maïs;
}

PAR M. ED. GRIFFON.

Depuis quelques années l'attention a été appelée sur les travaux de DE VRIEs ${ }^{1}$ concernant l'origine des plantes par mutation, c'est-à-dire par " production subite, et sans transitions, de formes nouvelles et stables dans les lignées pures d'espèces bien définies ". D'après le savant hollandais la cause de ces changements brusques agit sur peu d'espèces à la fois et d'une façon périodique; en outre elle est inconnue.

Depuis 1901 M. Blaringhem ${ }^{2}$ s'est employé à obtenir, en particulier chez le Maïs, des phénomènes de mutation à l'aide de traumatismes faits à une " époque convenable " du développement des plantes. Des mutilations méthodiques auraient provoqué brusquement l'apparition d'un grand nombre de formes stables dont beaucoup "sont tout à fait nouvelles en ce sens qu'on ne les avait jamais signalées jusqu'ici et que la plupart montrent des caractères aberrants pour l'espèce, le genre et mème la famille qui renferment la forme initiale ". Les travaux de l'auteur sur le Maïs et sur quelques autres plantes sauvages ou cultivées lui auraient permis d'esquisser dès 1907 " un programme de recherches dont la réalisation aboutirait à la création raisonnée et expérimentale d'espèces nouvelles ".

Mon but n'est pas, dans la présente communication de faire une critique d'ensemble de la mutation, de sa signification et de sa portée, ainsi que des causes qui la provoquent. Je veux me borner simplement à présenter quelques observations : $1^{\circ}$ sur les monstruosités florales du Maïs; $2^{\circ}$ sur les variétés nouvelles obtenues à la suite de traumatismes et dont les graines sont distribuées par les soins du Muséum d'Histoire naturelle (Service de la Culture). Ces observations ne consti-

1. DE VRies, Espèces et variètès, 1 vol., Paris, 1909.

2. Blaringhem, Mutation et traumatismes, 1 vol., Paris, 1907. (Thèse de Doctorat.) 
tuent à mes yeux qu'un document auquel, espérons-le, il viendra s'en joindre beaucoup d'autres, ce qui nous permettra de nous faire une idée plus précise sur la nature et l'importance des résultats obtenus jusqu'ici.

Car, il faut l'avouer, une grande confusion règne en ce moment au sujet 'de ces résultats dans le monde agricole et même dans le monde scientifique; cela tient en partie à ce que les travaux exécutés dans ces derniers temps en Suède, en Hollande et en France ont des conséquences à la fois théoriques et pratiques et que les unes, suivant la façon dont chacun de nous les apprécie, font souvent et à tort admettre ou rejeter les autres.

On crée, dit-on, des variétés nouvelles de Maïs et on les propose aux cultivateurs qui ont à en juger les mérites; ceux-ci peuvent être faibles ou nuls et la création des variétés demeurer cependant un fait biologique important; inversement, on peut n'avoir rien produit de nouveau, mais seulement séparé quelque chose de bon qui existait déjà. On sélectionne, comme à Svalöf, des Orges par la méthode généalogique et l'on arrive à pouvoir fournir aux cultivateurs des sortes pures qui donnent des produits estimés des brasseurs par leur grande homogénéité chimique et physiologique; c'est intéressant et fort utile; mais dans ce cas on n'a pas créé de formes réellement nouvelles, et la méthode de sélection usitée est connue non seulement à l'étranger, mais même en France où il semble bien qu'elle ait pris naissance et où elle est couramment employée ${ }^{1}$.

Il était absolument nécessaire de faire ces remarques au préalable; sans cela on exposerait les lecteurs non avertis - et ils sont nombreux - à se faire une opinion fausse sur la signification et la portée des résultats nouveaux ou soi-disant tels qui viennent d'être mis en avant.

\section{II}

J'arrive maintenant à l'examen des monstruosités que j'ai observées dans mon champ d'expériences de l'École de Grignon et à celui des variétés nouvelles obtenues par M. B LARINGHEM . Je rappelle que le Maïs, céréale d'origine américaine, est cultivé soit pour son fruit, soit comme fourrage. Il comporte un grand nombre de variétés ou espèces affines; plusieurs

1. Vilmorin (Ph. de), De la sélection des Semences (Bull. de la Soc. des Agriculteurs de France, 1907, p. 309). 
auteurs ont séparé de l'espèce linnéenne $Z$ ea Mays le $Z$. Curagua Mol. à feuilles denticulées, cultivé au Chili, le Z hirta Bon., de la Californie, à feuilles et glumes hérissées, à épillets moyens et supérieurs de l'épi màle sessiles, le $Z$. erythrolepis Bon., dẹs rives du Missouri, à graines aplaties et surtout à glumes et glumelles de l'épi femelle toujours colorées en rouge, le $Z$. rostrata, du Pérou, à graines terminées en bec, le $Z$. cryptoderma ( $Z$. tunicata Aug. St.-Hil.), du Paraguay, à glumes revêtant complètement le grain.

Les variétés de l'espèce type et des espèces voisines se classent pratiquement en Maïs à bec, Maïs sans bec à grains ovoïdes, allongés, aplatis (blancs, jaunes, jaune jaspé de rouge, rouges ou roses, violets). La variété qui a été étudiée par M. Blaringhem est le Maïs jaune de Pensylvanie ( $Z$ ea Mays pensylvanica Bon. $=Z$. M. vulgaris var. vulgata Körn. et Wern.). Ce Maïs est cultivé dans le Nord de la France comme fourrage.

En 1908 j'ai cultivé à Grignon toutes les variétés de Maïs de la maison Vilmorin afin d'étudier les anomalies florales qu'elles pourraient présenter. Aucun pied ne fut recépé ni blessé d'une façon quelconque; aucun ne portait trace de parasite connu, de charbon notamment ayant, comme on sait, la propriété de changer le sexe des fleurs atteintes. Le nombre des anomalies a été très élevé.

L'anomalie la plus fréquente consiste en épis latéraux ramifiés portant un grand nombre de grains avec ou sans fleurs mâles (Maïs perle : 2 pieds sur 12; King Philip : 5 pieds sur 12; Jaune hâtif à épis longś : 3 pieds sur 12; Maïs à bec : 1 pied sur 12; Rouge gros : 1 pied sur 12; Pop Korn : 2 sur 12; Cinquantino : 1 sur 12; Jaune gros : 2 sur 12; etc.). Cette anomalie est bien connue; elle a été signalée à maintes reprises; Boccone et Morison avaient même voulu en faire une espèce (Zea Mays polystachya); mais ce n'est qu'une monstruosité qui ne semble pas héréditaire. A la fin du xviri ${ }^{e}$ siècle un cultivateur de Pantin, SaInt-Genis ${ }^{1}$, l'a signalée avec d'autres qu'on a retrourées depuis, et il a constaté que les graines des épis ramifiés ont toujours produit des épis ordinaires. Je n'ignore pas, et je dirai cela une fois pour toutes, que la fécondation croisée est chose commune chez le Mais, que par conséquent il se peut qu'elle ait eu lieu dans l'expérience de cet agriculteur, que par suite les

1. Résultats de diverses cultures en l'an $\boldsymbol{V}$ dans un terroir près de Paris (Annales de l'Agriculture française, t. II, p. 368). 
graines aient donné dans la descendance le caractère ancien, dominant, c'est-à-dire la non-ramification de l'épi.

M. Blaringhem, rapprochant cette forme à épis ramifiés du Zea canina Watson ou "Maïs de Coyotte ", décrit ici mème par notre confrère M. Ph.fde VILmorin ${ }^{1}$, se croit autorisé à conclure que le genre Zea n'est qu'une forme monstrueuse du genre Euchlæna (Reana) née et propagée par les soins de l'homme. J'ai cultivé le Téosinte dans ma serre de Grignon; en outre, de deux graines de Zea canina, j'ai obtenu en 1907 deux pieds très beaux dont l'un a donné un épi ramifié. En 1908 j'ai semé trois graines parmi celles récoltées l'année précédente; j’ai eu trois plantes qui se sont très bien développées; elles étaient encore plus vigoureuses qu'en 1907; malheureusement elles n'ont pas fructifié.

On sait aujourd'hui que le Zea canina n'est qu'un hybride fertile dérivé du Téosinte et du Maïs, comme l'ÁEgilops speltæformis est un hybride fertile dérivé de l'Agilops ovata et du Blé. Or, pas plus qu'on admet comme établi que le Blé provient de l'A Egilops, on ne doit considérer comme démontré que le Maïs dérive du Téosinte. Il y a bien les épis ramifiés du Maïs; mais leur structure est différente de celle des épis du $Z$ ea canina, surtout en ce qui concerne les graines, lesquelles sont enchàssées dans une sorte de capsule soudée à sa voisine et ressemblent à un Maïs à bec. La forme hybride s'est maintenue chez moi sans disjonction en 1907, et certainement aucun cas de xénie, résultant de fécondation croisée, n'a pu se produire, étant données les conditions spéciales de ma culture en serre.

Quelquefois l'épi femelle des Maïs était ramifié sans présenter de fleurs mâles ou bien il n'était pas ramifié et présentait des fleurs mâles au sommet (King Philip blanc, 4 pieds sur 12; Jaune hâtif à épis longs, 1 sur 12; etc.). Un épi de Pop Korn présentait des fleurs mâles d'un côté et des fleurs femelles de l'autre; un épi de King Philip blanc portait de bas en haut, en alternance, des groupes de fleurs mâles et des groupes de fleurs femelles.

1. Vilmorin (Ph. de), Reana luxurians $\times$ Zea Mays (Bull. de la Soc. bot. de France, 1907, p. 39). 
Enfin des inflorescences mâles montraient quelques ovules à la base (Sucré nain hâtif).

Tous les épis anormaux se trouvaient sur des tiges latérales nées sans blessure; c'est du reste là un fait d'observation bien établi que de telles tiges sont souvent monstrueuses; il n'est donc pas étonnant qu'après le sectionnement de la tige principale, on obtienne des anomalies florales.

Toutefois le Jaune et le Blanc hâtif des Landes m'ont donné deux inflorescences mâles avec grains à la base sur la tige principale.

En tenant compte de tous les cas de monstruosités florales obtenues dans mes cultures de 1908, j'ai constaté que le King Philip blanc a eu 10 pieds monstrueux sur 10 , le K. P. brun 9 sur 12 , le Maïs perle 7 sur 12 , le Sucré ridé toujours vert 5 sur 12. Les variétés précoces n'ont pas tallé, sont restées chétives et sans variations, sauf le Maïs des Landes. En outre, des variétés très vigoureuses et sans talles, comme le Maïs de Cuzco et le Maïs Dent de cheval, n'ont pas donné d'anomalies.

Enfin, les épis monstrueux présentaient, comme on le remarque très souvent, une torsion marquée du pédoncule.

J'ai cultivé la même collection de Maïs en 1909 en utilisant encore des graines de la Maison Vilmorin et j’ai eu très peu de monstruosités, aussi bien sur les rejets des variétés américaines (King Philip, etc.) que sur les variétés indigènes; cela est dû certainement aux différences de conditions météorologiques, à Grignon, entre les deux années 1908 et 1909 .

Au cours de cette année 1909, j'ai sectionné la tige de plusieurs pieds (Jaune hâtif d'Auxonne, King Philip) et les rejets n'ont rien donné d'anormal.

J'ai semé des graines d'épis monstrueux récoltées en 1908. En particulier des graines d'une inflorescence femelle ramifiée de Jaune hâtif à épis longs : sur 8 pieds, 2 ont été monstrueux; l'un a donné un épi femelle ramifié et l'autre une panicule mâle avec graines à la base. Il est impossible de tirer de cette expérience une conclusion rigoureuse qu'elle ne comporte pas à cause du vicinisme possible d'abord, du fait que des anomalies semblables se produisent çà et là, sans cause apparente, avec la même fréquence, ensuite.

Une constatation que je dois faire sans plus tarder au sujet du vicinisme (faux atavisme dù à la fécondation croisée entre variétés cultivées dans le voisinage l'une de l'autre), c'est que, en 1808 et en 1909 , la xénie ne s'est pas manifestée. Tous les épis récoltés paraissaient purs et conformes au type et leurs graines mûres ont donné les années suivantes les variétés dont elles dérivaient. Au contraire en 1910 la xénie a été très fréquente; toutes les variétés ayant mùri (pensylvanica et ses dérivés, King Philip, Blanc des Landes, Rouge gros, Ridé hâtif, etc.) 
portaient des traces de l'influence du Maïs perle, si reconnaissable.

Au cours de cette même année 1910 j'ai recépé des pieds appartenant aux variétés pensylvanica, Auxonne, King Philip; sauf pour le second, la proportion des rejets formés a été la même que sans recépage, comme le montre le tableau ci-dessous :

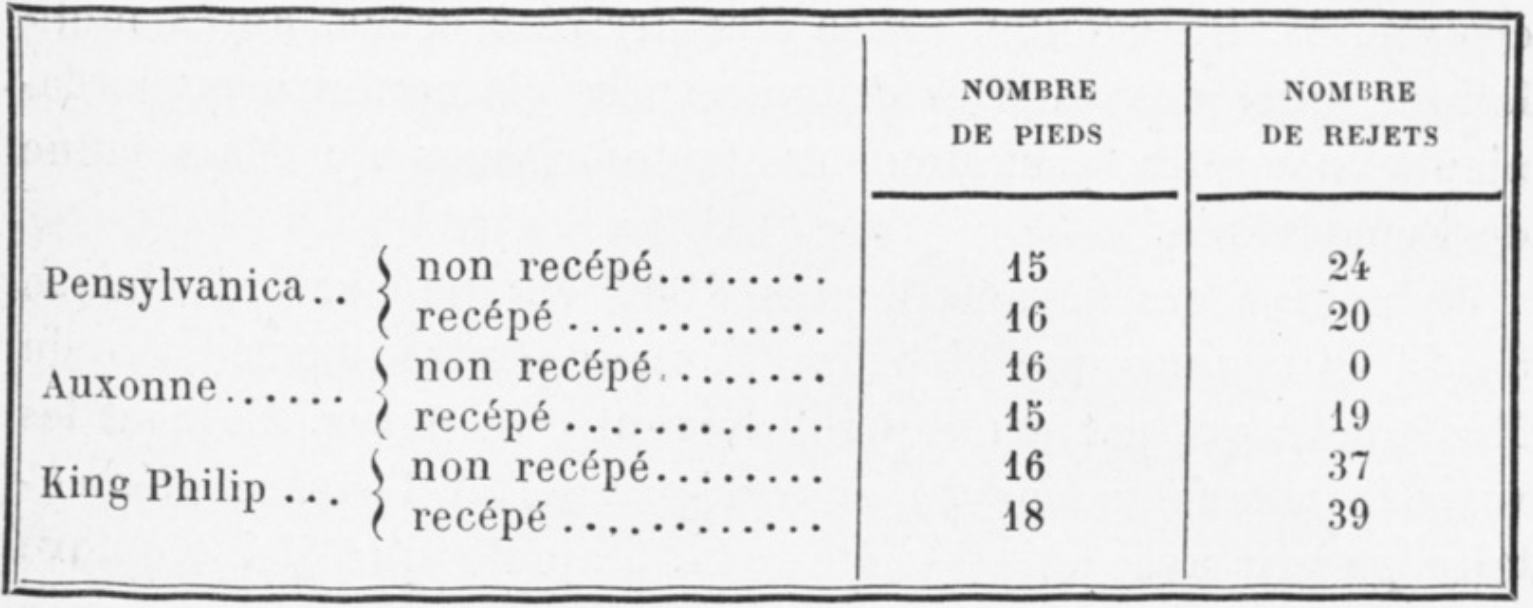

Les anomalies florales étaient les mêmes qu'en 1908 (épis femelles ramifiés avec ou sans fleurs mâles; panicules mâles portant des graines fertiles). Ce second type de monstruosités est apparu exclusivement sur rejets, alors que le premier se rencontrait, contrairement à ce qui s'est passé en 1908 et 1909 , aussi bien sur la tige principale que sur les pousses latérales (Auxonne, pensylvanica, Ridé hâtif).

Je n'ai pas obtenu les monstruosités décrites par M. BLARinghem sous le nom de forme Chou-fleur; mais cette variété apparait sur le Zea Mays tunicata que je n'ai pas encore cultivé. J'aurai l'occasion de m'en occuper prochainement.

On voit par tout ce qui précède combien est complexe et encore obscure cette question de l'origine et de l'hérédité des monstruosités chez le Maïs. Ce n'est pas en quelques années d'expériences qu'on peut l'élucider. M. Blaringhem et moi, nous avons obtenu ce que de nombreux auteurs ont trouvé avant nous et je ne vois pas que nous ayons apporté beaucoup de nouveau. M. Blaringhen reconnait (loc. cit., p. 179) qu'il a produit simplement des variétés instables (eversporting de Hugo DE V $\mathrm{RIEs),} \mathrm{que} \mathrm{le} \mathrm{Maïs} \mathrm{est} \mathrm{une} \mathrm{mauvaise} \mathrm{plante} \mathrm{pour} \mathrm{l'étude} \mathrm{de}$ l'hérédité, mais que le seul fait d'avoir pu récolter chaque année des individus ayant conservé les caractères anormaux des parents montre que la transmission est certaine. Quant à

T. LVII.

(SÉANCES) 39 
moi, sur ce point particulier, ma conviction est bien moins assurée et je considère le problème comme non encore résolu ${ }^{1}$.

\section{III}

Cependant, malgré les difficultés d'empêcher la fécondation croisée, M. BLaringhem pense être arrivé à créer, après mutilations, quelques variétés et une espèce élémentaire ou jordanienne nouvelles, parfaitement stables, issues du Maïs jaune de Pensylvanie.

Je cultive ces formes depuis 1907 en me servant chaque année de graines que distribue l'auteur par l'intermédiaire du Muséum et qui par conséquent doivent être pures. Ce sont les variétés pseudo-androgyna et semi-præcox et l'espèce élémentaire præcox avec une variété alba. J'ajoute que j'ai employé aussi, autant que la maturité me l'a permis, des graines récoltées dans mon champ d'expériences.

Le pseudo-androgyna présente, sur un certain nombre de grains des épis femelles, des étamines plus ou moins développées. Le semi-prær,ox serait plus précoce de trois semaines que le type; les pieds sont plus épais, plus trapus, de taille moins élancée, les épis latéraux gros et courts. Enfin, le præcox, plante grềle, basse, à nœuds courts, est plus hâtif que les précédents, ce qui a permis son isolement avec facilité; cette espèce élémentaire a donné une variété à grains blancs.

Faut-il considérer toutes ces formes nouvelles comme des variétés, sauf la dernière, ou bien faut-il voir dans toutes des espèces élémentaires, c'est là une affaire de point de vue qui conduirait à une discussion oiseuse.

Dès 1907 j'ai observé une petite différence de taille entre le type pensylvanica et les variétés pseudo-androgyna et semi-præcox, mais pas très marquée; par contre le præcox, jaune ou blanc, était bien nain. Les grains de semi-præcox récoltés à Grignon étaient plus arrondis, plus pleins que ceux du pseudo-androgyna de même provenance. Le pseudoandrogyna de Grignon avait les grains conformes au type primitif. Quant

1. M. Metchnikoff déclare qu'à côté de certains exemples de variations provoquées par l'homme et des parasites, il en existe d'autres où la cause intime échappe complètement. Il serait intéressant, dit-il, d'étudier si, dans ces cas, il ne s'agirait pas de parasitisme microscopique ou même invisible. (Revue scientifique, $1909,2^{\mathrm{e}}$ semestre, p. 548.) 
aux grains du præcox, ils étaient plus gros et plus uniformes comme taille que ceux du Muséum; enfin le præcox alba avait des grains jaunes et des grains blancs, ce qui peut être dû soit à l'atavisme, soit au vicinisme.

En 1908 j'ai semé, comparativement, des grains venant du Muséum et des grains récoltés à Grignon en 1907. Au 30 juillet les pensylvanica, pseudo-androgyna et semi-præcox des deux provenances ne présentaient aucune différence d'aspect; même observation pour le 22 septembre. Je les ai montrés à des personnes habituées à examiner des plantes cultivées, et toutes, non prévenues de ce qu'elles avaient sous les yeux, ont conclu qu'il n'y avait là qu'une seule variété de Maïs. Quant au præcox venu de graines de Grignon, il présentait 3 pieds presque identiques au type pensylvanica dont il dérive (vicinisme ou atavisme).

Sur un certain nombre de pieds de chaque forme il y a eu, en 1907 surtout, des anomalies semblables à celles qui ont déjà été décrites, et cela quelle que soit la provenance des graines, sauf cependant sur le præcox qui n'avait d'ailleurs pas de pousses latérales.

En 1909 mêmes résultats qu'en 1910 et, comme je l'ai dit plus haut, des grains d'inflorescence femelle ramifiée de semi-præcox ont donné 7 pieds normaux sur 8.

En 1910 j'ai opéré avec des grains du Muséum exclusivement. Les différences entre pensylvanica, pseudo-androgyna et semi-præcox m'ont paru nulles; une personne non au courant de l'expérience n'aurait pas songé à en chercher. On peut donc dire que les deux secondes formes ne sont pas héréditaires et que, à Grignon tout au moins ou dans des localités productrices des semences pures, elles sont retournées au type qui les avait engendrées. Le semi-præcox autofécondẻ m'a fourni des épis de $13 \mathrm{~cm}$. 5 et de $14 \mathrm{~cm}$. de long et, dans les mêmes conditions, les dimensions des épis du pensylvanica ont varié entre 10 et $14 \mathrm{~cm}$.

En ce qui concerne le pseudo-androgyna (autofécondé ou non), je dois dire que pour la première fois, en 1910, j'ai aperçu des étamines à la base des grains dans les épis femelles. Cette année, à Grignon, il y a eu beaucoup d'humidité jusqu'à la fin de juillet et, à partir de cette date jusqu'à la fin de septembre, sécheresse marquée; peut-être est-ce à cette condition météorologique spéciale que nous devons une bonne maturation des épis des variétés expérimentées et le développement des étamines chez les fleurs femelles. Mais ce caractère n'est pas spécial à la forme dérivée du Maïs de Pensylvanie; je l'ai retrouvé très nettement sur le pensylvanica type (autofécondé 
ou non), sur le semi-præcox (non autofécondé), sur le præcox (autofécondé), sur le Maïs perle, sur le King Philip, etc. C'est mème chez cette dernière variété que j'ai trouvé les étamines les plus grandes et les mieux conformées; les anthères faisaient saillie en entier et il était bien inutile d'enlever les grains pour les voir. Au reste ce phénomène de production d'étamines non seulement n'est pas spécial à une forme du Maïs de Pensylvanie, comme je viens de le montrer, mais il était connu. Penzig, dans son Pflanzenteratologie (p. 462) dit que des fleurs hermaphrodites (ovaire et 3 étamines) ont été signalées aussi bien dans l'épi femelle que dans la panicule mâle par Masters, Fermond, Kraft, Scott, Hempel. Ce doit ètre, en effet, très commun certaines années. Ce fait montre encore une fois combien il faut être prudent quand on parle de caractères nouveaux, de variétés nouvelles; une longue pratique des plantes cultivées est nécessaire pour ètre affirmatif, on ne saurait trop le répéter. Au début de l'année je tenais le caractère en question pour inexistant et maintenant j'ai la preuve qu'il a déjà été décrit et que dans certains cas il est commun.

J'ai autofécondé cette année plusieurs pieds de præcox jaune et blanc. J'ai eu de beaux épis avec le jaune, sans xénie par le Maïs perle qui a influencé tous les épis non autofécondés de mes cultures (pensylvanica et ses dérivés, Ridé nain hâtif, King Philip, Auxonne, etc.). Quant au præcox blanc, 10 pieds ont été autofécondés, 2 ne l'ont pas été ; malheureusement les épis femelles (pour quelle cause?) n'ont pas fructifié, de sorte qu'il m'a été impossible de résoudre le problème que je m'étais posé en 1907 à son sujet, à savoir si la présence de grains jaunes au milieu des blancs était un signe de dégénérescence par retour au type ou par vicinisme.

J'ajoute que les Maïs précoces, et surtout la variété à grains blancs, sont des plantes naines, dégénérées, comme il en apparaît de temps à autre sans traumatismes dans bien des espèces cultivées, que ce serait une grosse erreur de les considérer comme une acquisition utile pour les agriculteurs, au moins dans la région de Grignon, même au point de vue de la production des grains. Au reste il n'est pas plus précoce en cet endroit que le quarantain, le jaune hâtif d'Auxonne, le King Philip blanc et d'autres. 


\section{IV}

Les recherches précédentes montrent que, chez le Maïs et dans les conditions de mes cultures à l'Ecole de Grignon, il se produit sans blessures ni action parasitaire connue des anomalies d'inflorescences qui peuvent se ramener à deux types : $1^{\circ}$ ramification des épis latéraux avec ou sans fleurs mâles; $2^{\circ}$ transformation de fleurs mâles en fleurs femelles dans la panicule terminale plus ou moins déformée. De très nombreux faits analogues ont été signalés bien des fois au cours du siècle dernier. Certaines variétés (King Philip notamment) ont donné en 1908 beaucoup de pieds monstrueux (10 sur 12); les monstruosités se trouvaient en majeure partie sur des talles nées naturellement. En 1909 les mêmes variétés ont porté au contraire peu de monstruosités et en 1910 le nombre de ces dernières a été parfois aussi grand sur la tige principale que sur les talles.

L'apparition d'étamines à la base des caryopses de l'épi femelle n'est nullement une nouveauté; la forme pseudo-androgyna de M. BLaringhem est apparue à Grignon en 1910 sur plusieurs variétés; elle était du reste connue des tératologistes (Penzig, etc.).

Les épis latéraux ramifiés sont bien différents de ceux du Zea canina Watson, hybride de Téosinte (Reana luxurians) par Maïs, et rien n'autorise à considérer comme démontrée l'hypothèse maintes fois formulée d'après laquelle le Maïs serait une forme monstrueuse de Téosinte née et propagée par les soins de l'homme; on ne peut donc actuellement, pas plus qu'autrefois, reconstituer avec certitude l'évolution du genre $Z$ ea .

Les causes vraies de l'apparition des anomalies florales chez le Maïs sont loin d'être déterminées avec précision. Les troubles de nutrition engendrés par des blessures doivent agir là comme ailleurs où c'est connu depuis longtemps; mais il y a bien d'autres causes. Des monstruosités peuvent donc se rencontrer sur des pieds blessés sans que la mutilation ait joué un ròle dans leur production. Les anomalies florales nées à la suite de traumatismes ou autrement chez le Maïs ne paraissent pas héréditaires; il n'est pas encore suffisamment prouvé qu'elles 
puissent caractériser même des variétés instables (ou eversporting de DE VRIEs).

Quant aux variétés pseudo-androgyna et semi-præcox de M. Blaringheir, elles sont retournées au type Maïs jaune de Pensylvanie (Zea Mays pensylvanica).

Le Maïs précoce, né lui aussi du type précédent, est une pauvre acquisition, au moins dans la région de Grignon, où cependant cette année il a très bien mûri; de plus il est peut être possible qu'on puisse le faire retourner au type par une culture appropriée et il n'est même pas démontré que ce soit une nouveauté. Le præcox alba est plus chétif encore et il semble revenir à la forme à grains jaunes qui lui a donné naissance. Mais je ne suis pas encore en mesure de donner de ces deux cas de retour une preuve formelle; il faut aussi envisager l'action du vicinisme ou pseudo-atavisme. Les expériences continuent sur ce point.

En somme les travaux récents effectués sur le Maïs apportent un bien faible appoint à la théorie de la mutation; ils n'ont pas fail apparaître de caractères vraiment nouveaux; ils n'ont surtout nullement établi à mon sens que les traumatismes soient “ un facteur très important de l'évolution des formes végétales ». Il faudra donc autre chose pour renverser l'opinion que m'exprimait récemment dans une lettre un éminent horticulteur anglais, à savoir que, selon lui et ses confrères, " tout ce qu'on peut revendiquer pour la mutation a trait aux sous-variétés et non pas aux vraies espèces, que bien des races horticoles sont nées par transformations lentes, que la mutilation, qui fait parfois naître des monstruosités, est inapte à créer des variétés nouvelles $\gg$.

Je n'ai nullement la prétention, comme je le disais au début de cette Note, de porter un jugement d'ensemble sur les théories concernant la variation et l'origine des espèces. Il faut pour cela une longue pratique des plantes cultivées, une connaissance approfondie des formes sauvages : mon expérience personnelle est trop bornée; mais j'ai pensé qu'autant il est absurde de rejeter dédaigneusement comme faux tout système ne cadrant pas avec les idées qui nous sont familières, autant il est dangereux d'accepter sans les soumettre au contrôle de l'expérience, quand 
M. AUBERTOT. - Sur L'anatomie des rameaux polymorphes.

on le peut, des explications nouvelles devant avoir, au dire de leurs auteurs, de grandes conséquences pour la philosophie naturelle et la culture des plantes.

M. Lutz donne lecture de la communication ci-dessous :

\section{Sur l'anatomie comparée des rameaux poly- morphes chez quelques arbustes épineux de la famille des Rosacées;}

PAR M. AUBERTOT.

Les extrémités épineuses proprement dites ont été l'objet de recherches approfondies, surtout de la part de Mittmann ${ }^{1}$, puis de Lothelier ${ }^{2}$; nous n'avons pas l'intention d'y revenir. Nous voulons simplement montrer que les rameaux épineux, au moins chez les sujets étudiés, ne diffèrent pas seulement des rameaux ordinaires par leurs caractères morphologiques extérieurs, mais aussi par leur structure, et que la structure de l'épine terminale se trouve en quelque sorte anatomiquement annoncée dès la base du rameau qui la porte.

Nos recherches ont porté principalement sur les Cratægus Oxyacantha L. et Prunus spinosa L., et aussi sur des individus sauvages spinescents des Pirus communis L., Pirus Malus L. et Mespilus germanica $\mathbf{L}$.

Morphologie externe. - D'une manière générale, les rameaux épineux sont plus courts que les rameaux ordinaires, dirigés dans tous les sens, parfois étalés à angle droit (Prunus spinosa). Leurs feuilles sont plus petites, moins vigoureuses (Pirus, Prunus), à lobes plus échancrés (Cratægus). La réduction stipulaire est toujours très importante, souvent énorme : chez le Cratægus par exemple, les stipules, puissamment développées dans les rameaux normaux, sont atténuées à la base et rétrécies dans les rameaux épineux, quand elles nefont pas complètement défaut.

1. Mittmans, Beiträge zur Kenntniss der Anatomie der Pflanzenstacheln. Inaug. Dissert., Berlin, 1888.

2. LOTHELIER, Recherches anatomiques sur les Épines et les Aiguillons des Plantes. Thèse, Paris, 1893. 


\section{$2 \mathrm{BHL}$ Biodiversity Heritage Library}

Griffon, Édouard. 1910. "Observations et recherches expérimentales sur la variation chez le Maïs." Bulletin de la Société botanique de France 57, 604-615. https://doi.org/10.1080/00378941.1910.10832270.

View This Item Online: https://www.biodiversitylibrary.org/item/8679

DOI: https://doi.org/10.1080/00378941.1910.10832270

Permalink: https://www.biodiversitylibrary.org/partpdf/160902

\section{Holding Institution}

Missouri Botanical Garden, Peter H. Raven Library

\section{Sponsored by}

Missouri Botanical Garden

\section{Copyright \& Reuse}

Copyright Status: Public domain. The BHL considers that this work is no longer under copyright protection.

This document was created from content at the Biodiversity Heritage Library, the world's largest open access digital library for biodiversity literature and archives. Visit BHL at https://www.biodiversitylibrary.org. 